ARTICLE

Received 2 Feb 2015 | Accepted 27 Apr 2015 | Published 4 Jun 2015

\title{
High-performance ternary blend polymer solar cells involving both energy transfer and hole relay processes
}

\author{
Luyao Lu', Wei Chen ${ }^{2,3}$, Tao $\mathrm{Xu}^{1} \&$ Luping $\mathrm{Yu}^{1}$
}

\begin{abstract}
The integration of multiple materials with complementary absorptions into a single junction device is regarded as an efficient way to enhance the power conversion efficiency (PCE) of organic solar cells (OSCs). However, because of increased complexity with one more component, only limited high-performance ternary systems have been demonstrated previously. Here we report an efficient ternary blend OSC with a PCE of 9.2\%. We show that the third component can reduce surface trap densities in the ternary blend. Detailed studies unravel that the improved performance results from synergistic effects of enlarged open circuit voltage, suppressed trap-assisted recombination, enhanced light absorption, increased hole extraction, efficient energy transfer and better morphology. The working mechanism and high device performance demonstrate new insights and design guidelines for highperformance ternary blend solar cells and suggest that ternary structure is a promising platform to boost the efficiency of OSCs.
\end{abstract}

\footnotetext{
${ }^{1}$ Department of Chemistry and The James Franck Institute, The University of Chicago, 929 E 57th Street, Chicago, IIlinois 60637, USA. ${ }^{2}$ Division of Materials Science, Argonne National Laboratory, Argonne, Illinois 60439, USA. ${ }^{3}$ Institute for Molecular Engineering, The University of Chicago, 5747 South Ellis Avenue, Chicago, Illinois 60637, USA. Correspondence and requests for materials should be addressed to L.Y. (email: lupingyu@uchicago.edu).
} 
mpressive advances in bulk heterojunction (BHJ) polymer solar cells (PSCs) have been made via material synthesis, device designs, physical measurements and theoretical understanding, which lead to much improved power conversation efficiency (PCE; exceeding $10 \%$ in both single junction and tandem cells $)^{1-4}$. However, despite the improvements achieved, PCEs of the state-of-the-art PSCs are still limited by factors such as insufficient light harvesting and low charge carrier mobilities of the active layer materials, restraining maximum achievable short circuit current density $\left(J_{\mathrm{sc}}\right)$ in the devices. A major research effort in the field has been focused on developing new polymers with tunable optoelectronic properties, optimizing device morphology as well as interfacial engineering to enhance exciton dissociation and reduce charge recombination ${ }^{1,5-16}$.

Recently, the use of ternary structure-active layer is emerging as a promising strategy to enhance the performances in binary polymer:fullerene $\mathrm{BHJ}$ devices ${ }^{17-20}$. Compared with the binary counterpart, ternary blend PSCs contain one more electron donor or acceptor. In many successful cases, the option of materials as the third component is rather wide open. It could be a quantum $\operatorname{dot}^{21}$, a small molecule 22,23 or a polymer ${ }^{18,24,25}$, which offers considerable opportunities for solar cell optimization. In addition to a complementary absorption with the primary donor material in the solar spectrum and cascade energy levels between highest occupied molecular orbital (HOMO) and lowest unoccupied molecular orbital (LUMO) energy levels of the dominating donor polymer and fullerene acceptor to facilitate charge transport ${ }^{17,26}$, our recent results revealed that the ternary blend exhibits improved nanomorphology over the binary device by carefully selecting the third component used. The synergistic effects led to an inspiring PCE of $8.22 \%$ for the ternary blend PSCs ${ }^{27}$.

In this work, we use poly-3-oxothieno[3,4-d]isothiazole-1,1dioxide/benzodithiophene (PID2) ${ }^{28}$ as the additional donor material into binary PSCs based on poly[4,8-bis(5-(2ethylhexyl)thiophen-2-yl)benzo[1,2-b:4,5-b']dithiophene-co-3fluorothieno[3,4-b] thiophene-2-carboxylate] (PTB7-Th) ${ }^{29}$ and $[6,6]$-phenyl $\mathrm{C}_{71}$ butyric acid methyl ester $\left(\mathrm{PC}_{71} \mathrm{BM}\right)$. We achieve a PCE as high as $9.20 \%$ for the ternary device with $20 \%$ PID2 content without further device engineering. To our best knowledge, this is the best PCE reported for ternary PSCs to date. Detailed studies show that the enhancement results from a synergistic effect of enhanced light absorption, improved hole mobility, energy transfer between PID2 and PTB7-Th, reduced trap-assisted recombination and improved morphology. The results indicate that the mechanism of the enhancement is completely different from our recent work based on donor polymer PTB7 (ref. 27).

\section{Results}

Polymer design and characterization. Chemical structures of PID2, PTB7-Th and $\mathrm{PC}_{71} \mathrm{BM}$ used in the ternary structure are shown in Fig. 1a. HOMO and LUMO energy levels measured by cyclic voltammetry are $-5.12,-5.52$ and -6.00 and -3.60 , -3.50 and $-3.90 \mathrm{eV}$ for PTB7-Th, PID2 and $\mathrm{PC}_{71} \mathrm{BM}$, respectively, which are summarized in Fig. 1b. UV-vis absorption spectra of PTB7-Th and PID2 are presented in Fig. 1c. The maximum absorption for PTB7-Th was at $700 \mathrm{~nm}$ and for PID2, the absorption peak appeared at $610 \mathrm{~nm}$. The maximum absorption coefficients for PTB7-Th and PID2 are 28,339 and $31,951 \mathrm{~cm}^{-1}$ (Supplementary Fig. 1), respectively. The complementary absorption for the two polymers should be beneficial for charge generation inside the ternary devices. UV-vis absorption spectra of all ternary blend films with different PTB7-Th:PID2 weight ratios were measured and summarized in Fig. 1d. It is clear that incorporation of PID2 gradually increased film absorption strength from 400 to $625 \mathrm{~nm}$ while the film absorption capability from 625 to $800 \mathrm{~nm}$ was reduced. This is in consistent with the absorption spectra of PID2 and PTB7-Th in Fig. 1c and the absorption coefficients in Supplementary Fig. 1.

Solar cell characterization. The photovoltaic performance of the above ternary system was investigated on the basis of the following simple device structure: indium tin oxide a
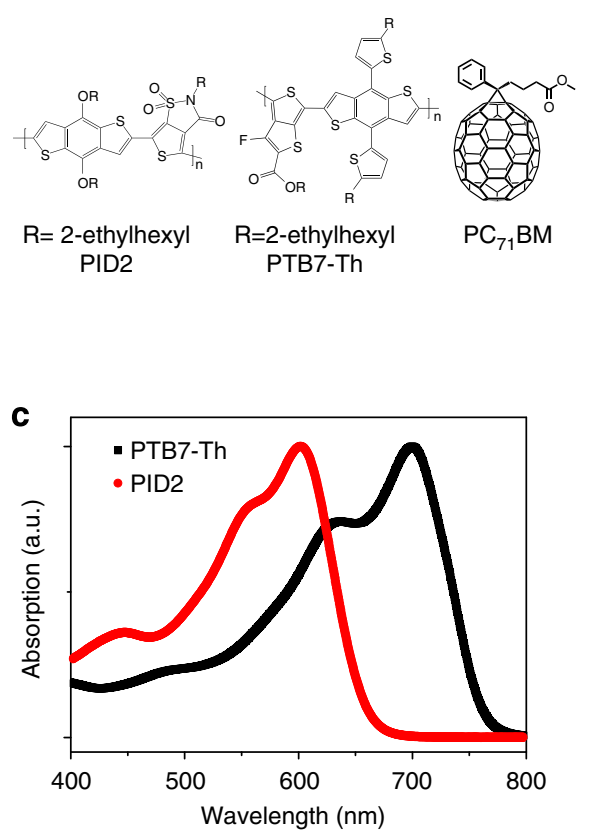

b
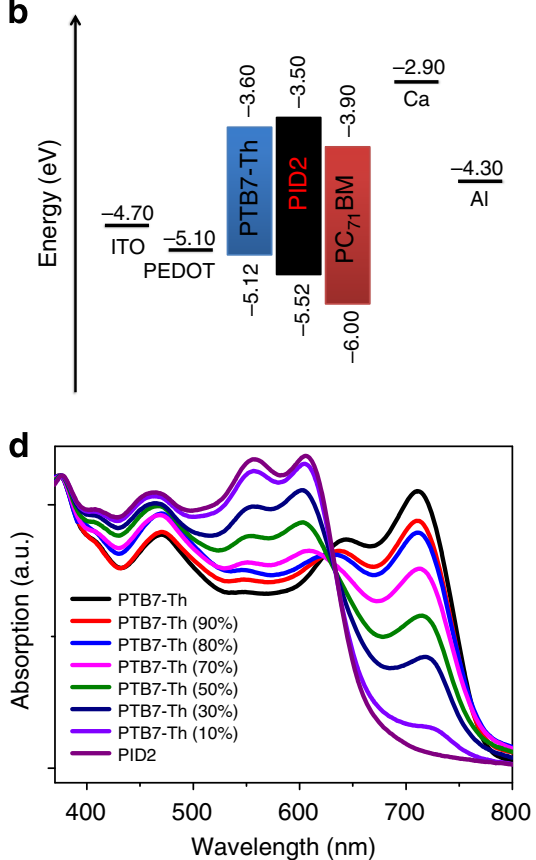

Figure 1 | Structures, electrical and optical properties of ternary blends. (a) Molecular structures of PID2, PTB7-Th and PC $C_{71} B M$. (b) Energy levels of materials used in the solar cell device. (c) UV-vis absorption spectra of PTB7-Th and PID2 in chloroform. (d) UV-vis absorption spectra of PTB7Th:PID2:PC ${ }_{71} B M$ films with different PTB7-Th:PID2 weight ratios. 
(ITO)/poly(3,4-ethylenedioxythiophene):poly(styrenesulphonate) (PEDOT:PSS)/PTB7-Th:PID2:PC ${ }_{71} \mathrm{BM} / \mathrm{Ca} / \mathrm{Al}$. The overall polymer:fullerene ratio was kept at 1:1.5 in this study. The corresponding photovoltaic performance parameters are summarized in Table 1. Figure 2a illustrates the representative current density versus voltage $(J-V)$ characteristics of devices with different PTB7-Th:PID2 weight ratios (0, 10 and 20\% PID2 incorporation) under simulated AM $1.5 \mathrm{G}$ illumination at $100 \mathrm{~mW} \mathrm{~cm}^{-2}$. Devices with different PID2 concentrations showed consistent thickness around $100 \mathrm{~nm}$. PTB7-Th:PC ${ }_{71} \mathrm{BM}$ control device started with a $J_{\mathrm{sc}}$ at $14.92 \mathrm{~mA} \mathrm{~cm}^{-2}$, an open circuit voltage $\left(V_{\mathrm{oc}}\right)$ at $0.75 \mathrm{~V}$, a fill factor $(\mathrm{FF})$ at $70.3 \%$ and a PCE of $7.88 \%$. By adding $10 \%$ of PID2 into the host binary blend, PCE was enhanced to $8.51 \%$ with a $J_{\mathrm{sc}}$ at $15.60 \mathrm{~mA} \mathrm{~cm}^{-2}$, a $V_{\mathrm{oc}}$ at $0.77 \mathrm{~V}$ and a $\mathrm{FF}$ at $70.9 \%$. Devices with $20 \%$ PID2 incorporation exhibited the best solar cell performance with a $J_{\text {sc }}$ at $16.68 \mathrm{~mA} \mathrm{~cm}^{-2}$, a $V_{\text {oc }}$ at $0.78 \mathrm{~V}$ and a $\mathrm{FF}$ at $70.8 \%$, resulting in a promising PCE of $9.20 \%$. This is more than $15 \%$ enhancement in

\section{Table 1 | Photovoltaic parameters for PTB7-Th:PID2:PC 71 BM} devices with different PID2 concentrations in PTB7-Th.

\begin{tabular}{lcccc}
$\begin{array}{l}\text { PTB7-Th:PID2: } \\
\text { PC }_{\mathbf{7 1}} \mathbf{B M}\end{array}$ & $\boldsymbol{J}_{\mathbf{s c}}\left(\mathbf{m A ~ c m}^{-\mathbf{2}}\right)$ & $\boldsymbol{V}_{\text {oc }} \mathbf{( V )}$ & $\mathbf{F F}(\%)$ & PCE (\%) \\
\hline 1:0:1.5 & 14.92 & 0.75 & 70.3 & 7.88 \\
$0.9: 0.1: 1.5$ & 15.60 & 0.77 & 70.9 & 8.51 \\
$0.8: 0.2: 1.5$ & 16.68 & 0.78 & 70.8 & 9.20 \\
$0.7: 0.3: 1.5$ & 14.04 & 0.79 & 71.6 & 7.95 \\
$0.5: 0.5: 1.5$ & 13.07 & 0.80 & 71.9 & 7.55 \\
$0.3: 0.7: 1.5$ & 11.50 & 0.83 & 70.0 & 6.68 \\
$0.1: 0.9: 1.5$ & 5.88 & 0.86 & 57.5 & 2.90 \\
$0: 1: 1.5$ & 5.29 & 0.86 & 44.3 & 2.01 \\
\hline
\end{tabular}

FF, fill factor; PCE, power conversion efficiency; PID2, poly-3-oxothieno[3,4-d]isothiazole-1,1dioxide/benzodithiophenepoly-3-oxothieno[3,4-d]isothiazole-1,1-dioxide/benzodithiophene.
PCE compared with the reference device. We would like to note that the 9.20\% PCE is the highest value reported for ternary blend PSCs so far. An average PCE of $8.97 \pm 0.16 \%$ was achieved over 10 identical devices at this condition with a mean $J_{\mathrm{sc}}$ at $16.22 \pm 0.35 \mathrm{~mA} \mathrm{~cm}^{-2}$, a $V_{\text {oc }}$ at $0.78 \pm 0.01 \mathrm{~V}$ and a FF at $70.8 \pm 0.14 \%$. PCE of the ternary device was still comparable to the reference cell even if PID2 content was enhanced to $50 \%$. FF remained larger than $70.0 \%$ even when PID2 content was increased to $70 \%$ and $V_{\text {oc }}$ increased monotonically with increased PID2 content at all ratios because of deeper HOMO energy levels of PID2 than PTB7-Th. This phenomenon was also observed for many other ternary systems reported ${ }^{20,30-32}$ and several possible mechanisms are proposed to explain the increased $V_{\mathrm{oc}}$, such as the electronic alloy charge-transfer state reported in ref. 33 and parallel-like BHJ concept demonstrated in ref. 18. The existence of co-crystals discussed later in the morphology part rules out the possibility of parallel tandem cell. It is likely that the increased $V_{\mathrm{oc}}$ with higher PID2 contents is from the electronic alloy chargetransfer state ${ }^{33}$ or the simple mixture of the two distinct chargetransfer states in PTB7-Th and PID2 (ref. 34). Further studies are needed to fully unravel the origin of $V_{\mathrm{oc}}$ changes in the future. Increasing the loading of PID2 to $90 \%$ led to dramatically decreased $J_{s c}$ and FF because of the poor performance of PID2 itself. We used devices with 10 and 20\% PID2 contents in the following discussions to clarify the effect of PID2 on the ternary system because of their much improved photovoltaic performance than the control device.

Changes in charge generation and transport properties. External quantum efficiency (EQE) spectra of the ternary devices are shown in Fig. 2b. EQE increased for devices with 10 and 20\% PID2 incorporation in a wide wavelength range from 350 to $750 \mathrm{~nm}$. The integrated $J_{\mathrm{sc}}$ values from EQE spectra for PTB7Th:PC ${ }_{71} \mathrm{BM}$ (1.0:1.5), PTB7-Th:PID2:PC $\mathrm{P}_{71} \mathrm{BM}$ (0.9:0.1:1.5) and PTB7-Th:PID2:PC ${ }_{71} \mathrm{BM}$ (0.8:0.2:1.5) devices are 14.52, 15.37 and

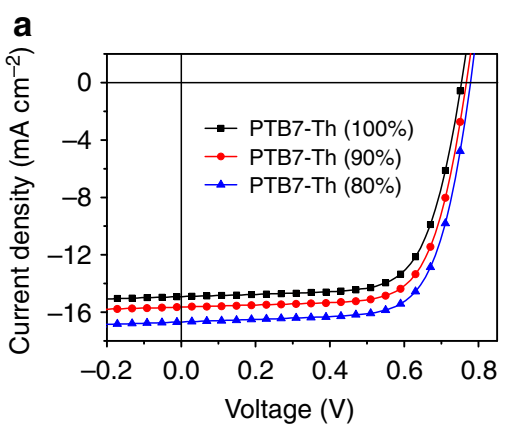

b
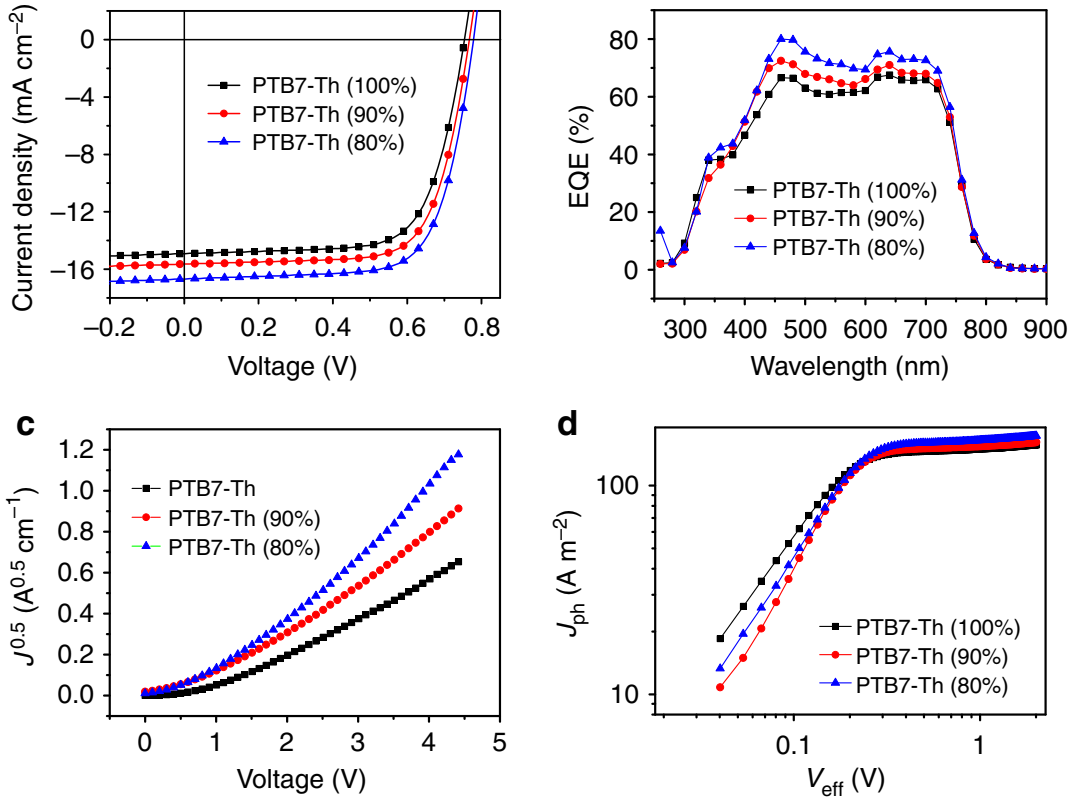

Figure 2 | Photovoltaic performance of the ternary devices. (a) Current density versus voltage characteristics of PTB7-Th:PID2:PC 71 BM with 0, 10 and 20\% PID2 content. (b) EQE curves for the ternary devices. (c) Hole mobility for the ternary system. (d) Photocurrent density versus effective voltage curves. 
$16.55 \mathrm{~mA} \mathrm{~cm}^{-2}$, respectively. This is similar to the $J_{\mathrm{sc}}$ values obtained from $J-V$ measurement (the error is within 3\%), indicating a good consistency of our solar cell measurements. By comparing with UV-vis absorption spectra in Fig. $1 \mathrm{~d}$, it is obvious that enhancement in EQE was not entirely because of absorption changes since addition of PID2 into the host blend actually decreased absorption intensity of PTB7-Th from 625 to $800 \mathrm{~nm}$. As shown in Fig. 1b, HOMO energy levels of PTB7-Th, $\mathrm{PID} 2$ and $\mathrm{PC}_{71} \mathrm{BM}$ constructed a cascade structure. As a result, PID2 could act as a hole relay to facilitate hole extraction from $\mathrm{PC}_{71} \mathrm{BM}$ to PTB7-Th after photoexcitation and charge separation $^{19,22,27}$. This is evidenced by the fact that EQE values in the 440 to $500 \mathrm{~nm}$ region were enhanced most significantly, where $\mathrm{PC}_{71} \mathrm{BM}$ absorbs.

Hole mobilities of the three devices were then evaluated by space charge-limited current method with the architecture: ITO/ PEDOT:PSS/PTB7-Th:PID2:PC ${ }_{71} \mathrm{BM} / \mathrm{MoO}_{3} / \mathrm{Au}$. Hole mobility increased from $3.58 \times 10^{-4} \mathrm{~cm}^{2} \mathrm{~V}^{-1} \mathrm{~s}^{-1}$ (PTB7-Th:PC ${ }_{71} \mathrm{BM}$, $1: 1.5)$ to $6.18 \times 10^{-4} \mathrm{~cm}^{2} \mathrm{~V}^{-1} \mathrm{~s}^{-1}$ (PTB7-Th:PID2:PC 71 BM, 0.9:0.1:1.5) and finally to $8.82 \times 10^{-4} \mathrm{~cm}^{2} \mathrm{~V}^{-1} \mathrm{~s}^{-1}$ (PTB7Th:PID2:PC ${ }_{71} \mathrm{BM}, 0.8: 0.2: 1.5$; Fig. $\left.2 \mathrm{c}\right)$. The averaged mobility values over 10 identical devices for the three conditions are $(3.33 \pm 0.22) \times 10^{-4} \mathrm{~cm}^{2} \mathrm{~V}^{-1} \mathrm{~s}^{-1} \quad(0 \%$ PID2 loading), $(5.96 \pm 0.32) \times 10^{-4} \mathrm{~cm}^{2} \mathrm{~V}^{-1} \mathrm{~s}^{-1} \quad(10 \%$ PID2 loading $)$ and $(8.46 \pm 0.30) \times 10^{-4} \mathrm{~cm}^{2} \mathrm{~V}^{-1} \mathrm{~s}^{-1}$ (20\% PID2 loading), respectively. The improved mobilities helped to partially explain the better $J_{\mathrm{sc}}$ in the ternary system.

More insight into the effect of PID2 on charge generation and dissociation process was gained by determining the saturation current density $\left(J_{\text {sat }}\right)$ and exciton dissociation probabilities $(P(E, T))$ of the ternary solar cells without and with 10 and $20 \%$ PID2 incorporation. Figure $2 \mathrm{~d}$ depicts photocurrent density $\left(J_{\mathrm{ph}}\right)$ versus effective voltage ( $\left.V_{\text {eff }}\right)$ characteristics for the three devices. $J_{\mathrm{ph}}$ is determined as $J_{\mathrm{ph}}=J_{\mathrm{L}}-J_{\mathrm{D}}$, where $J_{\mathrm{L}}$ and $J_{\mathrm{D}}$ are the photocurrent densities under one sun illumination and in the dark, respectively. $V_{\text {eff }}$ is determined as $V_{\text {eff }}=V_{0}-V_{\mathrm{a}}$, where $V_{0}$ is the voltage at which $J_{\mathrm{ph}}$ is 0 and $V_{\mathrm{a}}$ is the applied bias voltage ${ }^{35,36}$. Generally, it is assumed that all the photogenerated excitons are dissociated into free charge carriers at high $V_{\text {eff }}(2 \mathrm{~V}$ in this case) and $J_{\text {sat }}$ is then only limited by maximum exciton generation rate $\left(G_{\max }\right)$. As a result, $J_{\text {sat }}=q L G_{\max }$, where $q$ is elementary charge and $L$ is the thickness of the active layer ${ }^{36}$. $G_{\max }$ values for the three devices were $9.79 \times 10^{27} \mathrm{~m}^{-3} \mathrm{~s}^{-1}(0 \%$ PID2 loading, $\left.J_{\text {sat }}=156.8 \mathrm{~A} \mathrm{~m}^{-2}\right), 1.01 \times 10^{28} \mathrm{~m}^{-3} \mathrm{~s}^{-1}(10 \%$ PID2 loading, $J_{\text {sat }}=161.5 \mathrm{~A} \mathrm{~m}^{-2}$ ) and $1.08 \times 10^{28} \mathrm{~m}^{-3} \mathrm{~s}^{-1}$ $\left(20 \%\right.$ PID2 loading, $\left.J_{\text {sat }}=173.2 \mathrm{~A} \mathrm{~m}^{-2}\right)$, respectively. The average $G_{\max }$ values over 10 identical devices for the three conditions are $(9.69 \pm 0.01) \times 10^{27} \mathrm{~m}^{-3} \mathrm{~s}^{-1}$ (0\% PID2 loading), $(9.93 \pm 0.01) \times 10^{27} \mathrm{~m}^{-3} \mathrm{~s}^{-1} \quad(10 \% \quad$ PID2 loading $)$ and $(1.06 \pm 0.01) \times 10^{28} \mathrm{~m}^{-3} \mathrm{~s}^{-1}$ (20\% PID2 loading), respectively. The increased $G_{\max }$ with PID2 addition suggested increased overall exciton generations in the ternary devices. This is because of the complementary absorption with a small amount of PID2 incorporation and higher absorption coefficient of PID2 compared with PTB7-Th in the wavelength region below $625 \mathrm{~nm} . P(E, T)$ is determined from the ratio of $J_{\mathrm{ph}} / J_{\text {sat }}$ (ref. 37). $P(E, T)$ values at short circuit condition for the three devices were 94.8\% (0\% PID2 loading), 95.3\% (10\% PID2 loading) and $94.6 \%$ (20\% PID2 loading), respectively. The incorporation of $10 \%$ and $20 \%$ PID2 into PTB7-Th:PC ${ }_{71}$ BM did not change the overall charge dissociation process. This should be ascribed to the higher LUMO energy level of PID2 than PTB7-Th, which impeded the dissociation of excitons generated by PTB7-Th at PTB7-Th:PID2 interface.

The increased exciton generation from PID2 can enhance $J_{\mathrm{sc}}$ in two possible pathways. Excitons generated by PID2 could directly dissociate at the PID2:PC ${ }_{71} \mathrm{BM}$ interface and be collected by the electrodes. This is confirmed by the significantly enhanced EQE for ternary devices with (10 and 20\%) PID2 content from 500 to $625 \mathrm{~nm}$ where PID2 showed improved absorption. Alternatively, energy transfer from photoexcited PID2 to PTB7-Th occurs, followed by charge separation at the PTB7-Th:PC ${ }_{71} \mathrm{BM}$ interface. This explains the increased EQE from 625 to $750 \mathrm{~nm}$ for the ternary devices. Photoluminescence spectra of PTB7-Th only, PID2 only and PTB7-Th with 10 and 20\% PID2 films provide evidence for the energy transfer process (Fig. 3a). The photoluminescence spectra of these films were excited at $610 \mathrm{~nm}$, which was the maximum absorption of PID2. PID2 showed a broad emission peak from 650 to $800 \mathrm{~nm}$, which overlapped well with the absorption spectrum of PTB7-Th (Fig. 1c), making energy transfer between PID2 and PTB7-Th favourable. Figure 3a showed that by increasing the amount of PID2 in PTB7-Th, emission of PTB7-Th increased continuously while emission of PID2 was diminished. Although the PID2:PC ${ }_{71} \mathrm{BM}$ device only gave a $P(E, T)$ value at $65.6 \%$, energy transfer from PID2 to

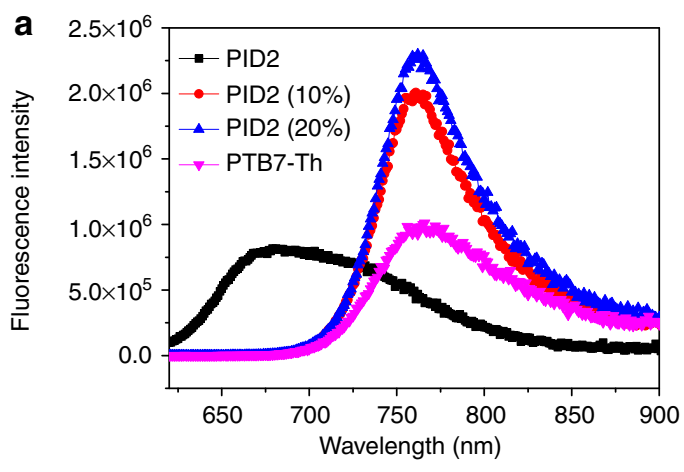

b

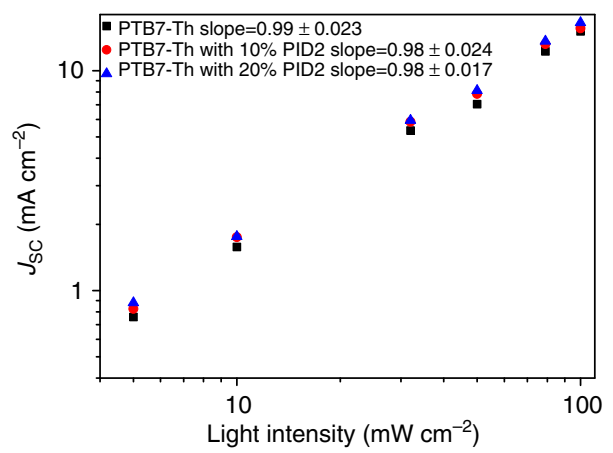

C

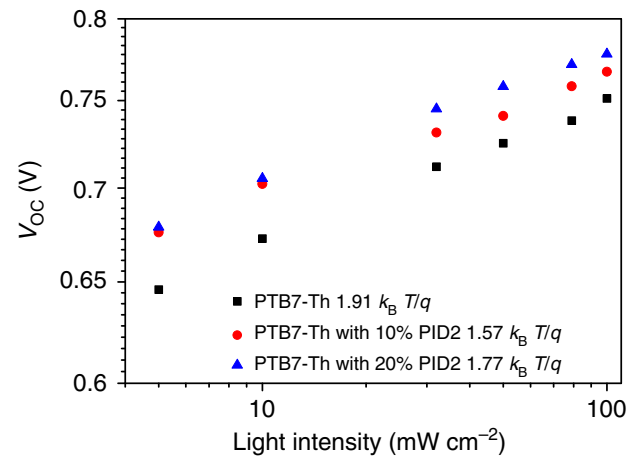

Figure 3 | Photoluminescence and recombination study of the ternary devices. (a) Photoluminescence spectra of PID2:PTB7-Th:PID2 (0.9:0.1, 0.8:0.2) and PTB7-Th films excited at $610 \mathrm{~nm}$. (b) Dependence of $J_{\mathrm{sc}}$ on light intensity for the ternary system. (c) Dependence of $V_{\text {oc }}$ on light intensity for the ternary system. 
PTB7-Th helped exciton dissociation because of a high $P(E, T)$ value at $94.8 \%$ for $\mathrm{PTB} 7-\mathrm{Th}: \mathrm{PC}_{71} \mathrm{BM}$ device.

Charge recombination dynamics. The dependence of $J_{\mathrm{sc}}$ and $V_{\mathrm{oc}}$ at various light intensities offers deeper insight into the influence of PID2 on recombination process in our ternary system. In principle, $J_{\mathrm{sc}}$ shows a power-law dependence on light intensity for organic solar cells, which can be expressed as $J_{\mathrm{sc}} \propto\left(P_{\text {light }}\right)^{S}$ (refs 38,39 ). Here $P_{\text {light }}$ is light intensity and $S$ is the exponential factor. Weak bimolecular recombination in the device would result in a linear dependence of $J_{\mathrm{sc}}$ on light intensity with $S$ value close to 1 . Figure 3b illustrates $J_{\text {sc }}$ as a function of $P_{\text {light }}$ for PTB7Th:PC ${ }_{71} \mathrm{BM}$ (1:1.5), PTB7-Th:PID2:PC ${ }_{71} \mathrm{BM} \quad(0.9: 0.1: 1.5)$ and PTB7-Th:PID2: $\mathrm{PC}_{71} \mathrm{BM}$ (0.8:0.2:1.5) devices. The extracted $S$ values are $0.99,0.98$ and 0.98 for the three devices, respectively. The similar $S$ values indicated that low loading of PID2 has negligible impact on bimolecular recombination process for the ternary system. This should be attributed to the fact that the control device already exhibited very weak bimolecular recombination with an $S$ value at 0.99 .

Figure $3 \mathrm{c}$ shows the relationship between $V_{\text {oc }}$ and $P_{\text {light }}$ in our ternary devices. The slope of $V_{\mathrm{oc}}$ versus $\log \left(P_{\text {light }}\right)$ helps us to determine the degree of trap-assisted recombination in the devices. A slope at $k_{\mathrm{B}} T / q$ implies that bimolecular recombination is the dominating mechanism, where $k_{\mathrm{B}}$ is Boltzmann's constant, $T$ is temperature and $q$ is elementary charge. As for trap-assisted or Shockley-Read-Hall recombination, a stronger dependence of $V_{\text {oc }}$ on light intensity with a slope of $2 k_{\mathrm{B}} T / q$ is observed ${ }^{38-40}$. In our cases, the PTB7-Th:PC ${ }_{71} \mathrm{BM}$ device showed a slope of 1.91 $k_{\mathrm{B}} T / q$ while for PTB7-Th:PID2:PC ${ }_{71} \mathrm{BM}(0.9: 0.1: 1.5)$ and PTB7Th:PID2:PC ${ }_{71} \mathrm{BM}$ (0.8:0.2:1.5) devices, smaller slopes of 1.57 and $1.77 k_{\mathrm{B}} T / q$ were attained. The results indicated that addition of PID2 into the PTB7-Th:PC $\mathrm{P}_{71} \mathrm{BM}$ blend reduced interfacial surface trap densities between the active layer materials in the devices, suppressed trap-assisted recombination and contributed to enhanced $J_{\mathrm{sc}}$.

Morphology characterization. Influence of PID2 on the morphology of the ternary system was determined by grazing incidence wide-angle X-ray scattering (GIWAXS) and resonant soft X-ray scattering (RSoXS). In the two-dimensional (2D) GIWAXS pattern for each individual polymer film (Supplementary Fig. 2), a broad arc-like scattering arises from the Bragg diffraction of periodic PTB7-Th layers $q_{\mathrm{y}}=0.301 \pm 0.009 \AA^{-1}$ and a distinct out-of-plane $\pi-\pi$ stacking peak appears at $q_{\mathrm{z}}=1.60 \pm 0.06 \AA^{-1}$, suggesting the preferential face-on conformation, whereas the PID2 bilayer is observed to order in a preferential edge-on orientation with the Bragg diffraction of periodic PID2 layers at $q_{\mathrm{y}}=0.346 \pm 0.009 \AA^{-1}$ and two off-axis scattering spots located at $( \pm 0.27,0.38) \AA^{-1}$, similar to the observation in PCDTBT ${ }^{41}$.
The 2D GIWAXS patterns also exhibit a scattering peak at $q_{\mathrm{y}}$ and $q_{\mathrm{z}} \sim 1.3-1.4 \AA^{-1}$ corresponding to the Bragg diffraction of $\mathrm{PC}_{71} \mathrm{BM}$. Because the full-width at half-maximum (FWHMs) of scattering peak correlates to the nanocrystallite size $^{42,43}$, the narrower FWHM of the PID2 layering peak indicates that PID2 in the binary films when blended with $\mathrm{PC}_{71} \mathrm{BM}$ could form larger nanocrystallite sizes than PTB7-Th (Table 2). It is intriguing that, when PTB7-Th and PID2 are blended together, they are not separated with each other, but form co-crystals, as evidenced by the changes in GIWAXS peak positions and their corresponding FWHMs of periodic PTB7-Th and PID2 layers (Supplementary Fig. 3 and Table 2). By incorporating a small amount of PID2 polymers into PTB7-Th:PC ${ }_{71} \mathrm{BM}$ blend films (PTB7Th:PID2:PC ${ }_{71} \mathrm{BM}, \quad 0.9: 0.1: 1.5$ and PTB7-Th:PID2:PC ${ }_{71} \mathrm{BM}$, 0.8:0.2:1.5), the PID2 polymers had little influence on the cocrystal structures of polymers, which remain similar to PTB7-Th polymers except a slight decrease in the nanocrystallite sizes. Meanwhile, the incorporation of PID2 polymers into the ternary films increases the $\mathrm{PC}_{71} \mathrm{BM}$ nanocrystallite sizes. On further introducing PID2 polymers (PTB7-Th:PID2: $\mathrm{PC}_{71} \mathrm{BM}, 0.3: 0.7: 1.5$ ), a new scattering peak occurs at $q_{\mathrm{y}}=0.316 \pm 0.009 \AA^{-1}$ in between the scattering of PTB7-Th and PID2 layers, rather than two individual peaks, indicating the intermixing of PTB7-Th and PID2 at the molecular level. The formation of co-crystals would facilitate energy transfer between PTB7-Th and PID2 since the rate constant of fluorescence resonance energy transfer is proportional to $(1 / r)^{6}$, where $r$ is the distance between PTB7-Th and PID2. In addition, the growth of larger $\mathrm{PC}_{71} \mathrm{BM}$ nanocrystallites could benefit charge transport within $\mathrm{PC}_{71} \mathrm{BM}$ domains.

Besides the molecular ordering, another important morphological factor that will determine the photovoltaic performance is how these localized molecular crystals and aggregates form phaseseparated domains in the BHJ blend. Figure 4a,b shows the RSoXS profiles (open symbols), the calculated scattering intensities, $I(q)$, (solid lines) and their corresponding pair distance distribution functions (PDDFs), $P(r)$, of the ternary PTB7Th:PID2:PC ${ }_{71} \mathrm{BM}$ BHJ films, respectively. These ternary PTB7Th:PID2:PC 71 BM BHJ films show hierarchical nanomorphologies at multiple length scales, in consistence with the previous observations in PTB7: $\mathrm{PC}_{71} \mathrm{BM}$ (ref. 44). As indicated by the zero crossings of $P(r)$ (Fig. $4 c, \mathrm{~d}$ ), the PID2:PC $\mathrm{P}_{71} \mathrm{BM}$ (1:1.5) film exhibits much larger domain sizes than the PTB7-Th:PC ${ }_{71} B M$ (1:1.5) film, which correlates with their unsatisfied device performance. When incorporating a small amount of PID2 polymers into PTB7-Th:PC ${ }_{71} \mathrm{BM}$ films (PTB7-Th:PID2:PC ${ }_{71} \mathrm{BM}$, 0.9:0.1:1.5 and PTB7-Th:PID2:PC $\left.{ }_{71} \mathrm{BM}, 0.8: 0.2: 1.5\right)$, such hierarchical nanostructural characteristics are retained with reduced domain sizes. A kink of $P(r)$ at the length scales of tens of nanometres indicates that the fine domains inside phaseseparated domains with hundreds of nanometres scale in these

Table 2 | Summary of 2D GIWAXS parameters of PTB7-Th:PID2:PC ${ }_{71} B M$ ternary systems.

\begin{tabular}{|c|c|c|c|c|c|c|}
\hline PTB7-Th:PID2:PC ${ }_{71}$ BM & Peak at $q_{y}\left(\AA^{-1}\right)$ & FWHM $\left(\AA^{-1}\right)$ & Peak at $q_{y}\left(\AA^{-1}\right)$ & FWHM $\left(\AA^{-1}\right)$ & Peak at $q_{z}\left(\AA^{-1}\right)$ & FWHM $\left(\AA^{-1}\right)$ \\
\hline $1: 0: 1.5$ & $0.301 \pm 0.009$ & 0.069 & $1.35 \pm 0.06$ & 0.24 & $1.37 \pm 0.06$ & 0.29 \\
\hline $0.9: 0.1: 1.5$ & $0.301 \pm 0.009$ & 0.074 & $1.35 \pm 0.06$ & 0.25 & $1.36 \pm 0.06$ & 0.32 \\
\hline $0.8: 0.2: 1.5$ & $0.306 \pm 0.009$ & 0.073 & $1.33 \pm 0.06$ & 0.20 & $1.36 \pm 0.06$ & 0.26 \\
\hline $0.5: 0.5: 1.5$ & $0.303 \pm 0.009$ & 0.049 & $1.29 \pm 0.06$ & 0.21 & $1.33 \pm 0.06$ & 0.30 \\
\hline $0.3: 0.7: 1.5$ & $0.316 \pm 0.009$ & 0.037 & $1.32 \pm 0.06$ & 0.21 & $1.35 \pm 0.06$ & 0.31 \\
\hline 0.1:0.9:1.5 & $0.337 \pm 0.009$ & 0.032 & $1.31 \pm 0.06$ & 0.23 & $1.35 \pm 0.06$ & 0.31 \\
\hline
\end{tabular}

2D, two-dimensional; FWHM, full-width at half-maximum. 

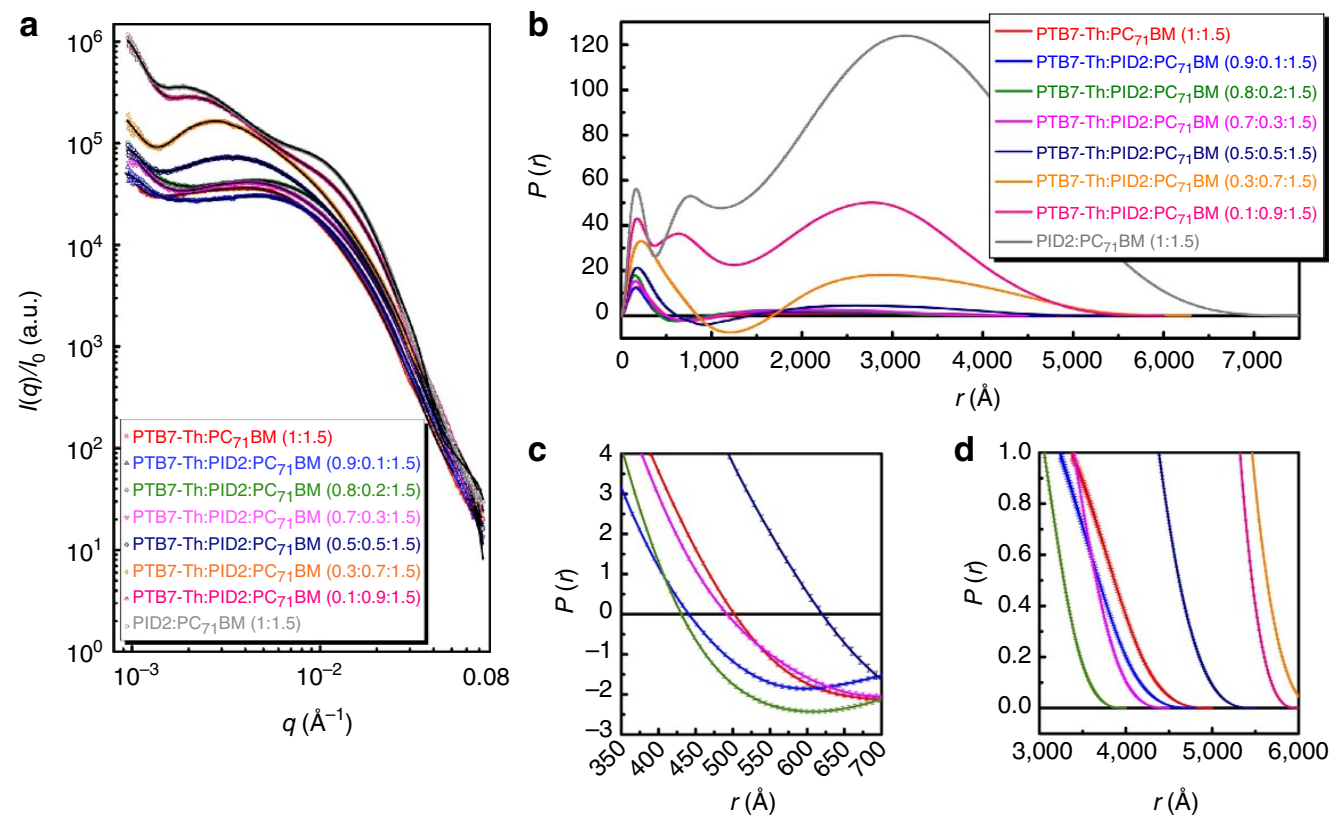

Figure 4 | RSoXS characterization of the ternary films. (a) RSoXS profiles (open symbols), calculated $I(q)$ (solid lines), and (b) corresponding $P(r)$ of PTB7-Th:PID2:PC ${ }_{71} B M$ ternary systems. (c,d) The enlarged regimes of $P(r)$ at the range of $r=350-700 \AA$ and $3,000-6,000 \AA$, respectively.

two ternary systems are even smaller than those of PTB7$\mathrm{Th}: \mathrm{PC}_{71} \mathrm{BM}$ (1:1.5). These smaller domains would increase the area of the interfaces between polymer donors and fullerene acceptors, thus promoting exciton dissociation and leading to improved performance. However, because of the poor performance of PID2, overall exciton dissociation probabilities in the ternary blends at 10 and $20 \%$ of PID2 incorporation are similar to that in the PTB7-Th:PC $\mathrm{PC}_{71} \mathrm{BM}$ binary blend. On further increasing the amount of PID2 polymers, larger domains are formed in the ternary blend films and, thereby, result in decreased device efficiency at high PID2 contents. Considering the complicated underlying working mechanism of the ternary $\mathrm{BHJ}$ solar cell, morphological characterization helps us to understand the tendency of performance change as a function of PID2 content in our devices.

\section{Discussion}

A highly efficient ternary structure PSC is developed by adding $\mathrm{PID} 2$ as the additional donor polymer into the PTB7-Th:PC $\mathrm{P}_{71} \mathrm{BM}$ binary device. A PCE of $9.20 \%$ was achieved with $20 \%$ of PID2 incorporation, which is the highest PCE reported for ternary PSCs. $V_{\text {oc }}$ of the ternary blends was enhanced with increased PID2 content because of the deeper HOMO energy levels of PID2 compared with PTB7-Th. A high FF at 70.0\% was maintained with PID2 content up to $70 \%$. In addition, the combined effects of a complementary light harvesting, efficient energy transfer from PID2 to PTB7-Th, enhanced hole mobility, reduced trap-assisted recombination, increased interfacial areas and better crystallinity of PCBM dramatically improved $J_{s c}$ in the ternary systems. Our results demonstrate that the use of ternary structure helps to re-evaluate the potential of many low-performance donor polymers and is a promising way towards developing high-performance PSCs.

\section{Methods}

Device fabrication. PTB7-Th and PID2 are synthesized according to previous procedures ${ }^{28,29}$. The number-averaged molecular weight of PTB7-Th and PID2 are 15.7 and $12.3 \mathrm{~kg} \mathrm{~mol}^{-1}$ with the dispersity index at 2.9 and 2.6 , respectively. PTB7Th, PID2 and $\mathrm{PC}_{71} \mathrm{BM}$ were dissolved in chlorobenzene and 1, 8-diodooctane $(97: 3, v / v)$. The overall donor polymer concentration (with or without PID2) was kept constant at $10 \mathrm{mg} \mathrm{ml}^{-1}$. The mixed solution was heated at $90^{\circ} \mathrm{C}$ overnight. ITO glasses were purchased from thin film devices and sonicated in water, acetone and isopropyl alcohol for $15 \mathrm{~min}$ each, followed by ultraviolet ozone irradiation for 60 min. Later, an $\sim 40$-nm-thick PEDOT:PSS layer was spin-coated at 6,000 r.p.m. on ITO glasses and dried at $80^{\circ} \mathrm{C}$ in oven for $30 \mathrm{~min}$. Polymer:PCBM solutions were spin-coated with the as-prepared solutions at 2,000 r.p.m. inside glove box. $\mathrm{Ca}(20 \mathrm{~nm})$ and $\mathrm{Al}(80 \mathrm{~nm})$ cathodes were thermal-evaporated.

Solar cell characterization. A solar simulator with a xenon arc lamp (Oriel model 69920) was used to provide 1-sun, AM 1.5G irradiation for the measurement of current density-voltage characteristics. Light intensity of the measurement was calibrated with an NREL-certified monocrystaline silicon reference cell (Newport, $91150 \mathrm{~V})$. The effective area of the $J-V$ measurement was determined by masks with well-defined area of $3.14 \mathrm{~mm}^{2}$. UV-vis spectra were taken from a UV-2401PC model UV-vis spectrophotometer. EQE was measured with a 250-W Quartz Tungsten Halogen (QTH) lamp as the light source.

Morphology characterization. GIWAXS measurements were conducted at the 8ID-E beamline ${ }^{45}$ at the Advanced Photon Source, Argonne National Laboratory using X-rays with a wavelength of $\lambda=1.6868 \AA$ and a beam size of $\sim 200 \mu \mathrm{m}(\mathrm{h})$ and $20 \mu \mathrm{m}(\mathrm{v})$. RSoXS measurements were achieved at beamline 11.0.1.2 (ref. 46) at the Advanced Light Source, Lawrence Berkeley National Laboratory. Details of GIWAXS and RSoXS measurements can be found in Supplementary Methods.

\section{References}

1. Li, G., Zhu, R. \& Yang, Y. Polymer solar cells. Nat. Photonics 6, 153-161 (2012).

2. He, Z. et al. Enhanced power-conversion efficiency in polymer solar cells using an inverted device structure. Nat. Photonics 6, 591-595 (2012).

3. You, J. et al. A polymer tandem solar cell with $10.6 \%$ power conversion efficiency. Nat. Commun. 4, 1446 (2013).

4. Liu, Y. et al. Aggregation and morphology control enables multiple cases of high-efficiency polymer solar cells. Nat. Commun. 5, 5293 (2014).

5. Son, H. J., He, F., Carsten, B. \& Yu, L. Are we there yet? Design of better conjugated polymers for polymer solar cells. J. Mater. Chem. 21, 18934-18945 (2011).

6. Lu, L. \& Yu, L. Understanding low bandgap polymer PTB7 and optimizing polymer solar cells based on it. Adv. Mater. 26, 4413-4430 (2014).

7. Peet, J. et al. Efficiency enhancement in low-bandgap polymer solar cells by processing with alkane dithiols. Nat. Mater. 6, 497-500 (2007).

8. Lee, J. K. et al. Processing additives for improved efficiency from bulk heterojunction solar cells. J. Am. Chem. Soc. 130, 3619-3623 (2008).

9. Liang, Y. et al. For the bright future-bulk heterojunction polymer solar cells with power conversion efficiency of 7.4\%. Adv. Mater. 22, E135-E138 (2010).

10. Dou, L. et al. A selenium-substituted low-bandgap polymer with versatile photovoltaic applications. Adv. Mater. 25, 825-831 (2013). 
11. You, J. et al. Metal oxide nanoparticles as an electron-transport layer in highperformance and stable inverted polymer solar cells. Adv. Mater. 24, 5267-5272 (2012).

12. He, Z. et al. Simultaneous enhancement of open-circuit voltage, short-circuit current density, and fill factor in polymer solar cells. Adv. Mater. 23, 4636-4643 (2011).

13. Son, H. J. et al. Synthesis and photovoltaic effect in dithieno[2,3-d:2', 3'-d']Benzo[1,2-b:4,5-b'] dithiophene-based conjugated polymers. Adv. Mater. 25, 838-843 (2013)

14. Guo, X. et al. Polymer solar cells with enhanced fill factors. Nat. Photonics 7, 825-833 (2013).

15. Cabanetos, C. et al. Linear side chains in Benzo[1,2-b:4,5-b']dithiopheneThieno[3,4-c]pyrrole-4,6-dione polymers direct self-assembly and solar cell performance. J. Am. Chem. Soc. 135, 4656-4659 (2013).

16. Zou, J., Li, C.-Z., Chang, C.-Y., Yip, H.-L. \& Jen, A. K. Y. Interfacial engineering of ultrathin metal film transparent electrode for flexible organic photovoltaic cells. Adv. Mater. 26, 3618-3623 (2014).

17. Ameri, T., Khoram, P., Min, J. \& Brabec, C. J. Organic ternary solar cells: a review. Adv. Mater. 25, 4245-4266 (2013).

18. Yang, L., Zhou, H., Price, S. C. \& You, W. Parallel-like bulk heterojunction polymer solar cells. J. Am. Chem. Soc. 134, 5432-5435 (2012).

19. Huang, J.-S. et al. Polymer bulk heterojunction solar cells employing Forster resonance energy transfer. Nat. Photonics 7, 479-485 (2013).

20. Cheng, P., Li, Y. \& Zhan, X. Efficient ternary blend polymer solar cells with indene-C60 bisadduct as an electron-cascade acceptor. Energy Environ. Sci. 7, 2005-2011 (2014).

21. Itskos, G. et al. Optical properties of organic semiconductor blends with nearinfrared quantum-dot sensitizers for light harvesting applications. Adv. Energy Mater. 1, 802-812 (2011).

22. Cha, H. et al. Complementary absorbing star-shaped small molecules for the preparation of ternary cascade energy structures in organic photovoltaic cells. Adv. Funct. Mater. 23, 1556-1565 (2013).

23. Zhang, Y. et al. Synergistic effect of polymer and small molecules for high-performance ternary organic solar cells. Adv. Mater. 27, 1071-1076 (2014).

24. Ameri, T. et al. Performance enhancement of the P3HT/PCBM solar cells through NIR sensitization using a small-bandgap polymer. Adv. Energy Mater. 2, 1198-1202 (2012).

25. Koppe, M. et al. Near IR sensitization of organic bulk heterojunction solar cells: towards optimization of the spectral response of organic solar cells. Adv. Funct. Mater. 20, 338-346 (2010).

26. Yang, L., Yan, L. \& You, W. Organic solar cells beyond one pair of donoracceptor: ternary blends and more. J. Phys. Chem. Lett. 4, 1802-1810 (2013)

27. Lu, L., Xu, T., Chen, W., Landry, E. S. \& Yu, L. Ternary blend polymer solar cells with enhanced power conversion efficiency. Nat. Photonics 8, 716-722 (2014).

28. $\mathrm{Xu}, \mathrm{T}$. et al. Tuning the polarizability in donor polymers with a thiophenesaccharin unit for organic photovoltaic applications. Adv. Funct. Mater. 24, 3432-3437 (2014).

29. Yu, L., Liang, Y. \& He, F. Semiconducting polymers. Patent WO 2013116643 A1 (2013).

30. Khlyabich, P. P., Burkhart, B. \& Thompson, B. C. Compositional dependence of the open-circuit voltage in ternary blend bulk heterojunction solar cells based on two donor polymers. J. Am. Chem. Soc. 134, 9074-9077 (2012).

31. Khlyabich, P. P., Burkhart, B. \& Thompson, B. C. Efficient ternary blend bulk heterojunction solar cells with tunable open-circuit voltage. J. Am. Chem. Soc. 133, 14534-14537 (2011).

32. Kang, T. E. et al. Photoinduced charge transfer in donor-acceptor (DA) copolymer: fullerene bis-adduct polymer solar cells. ACS Appl. Mater. Interfaces 5, 861-868 (2013)

33. Street, R. A., Davies, D., Khlyabich, P. P., Burkhart, B. \& Thompson, B. C. Origin of the tunable open-circuit voltage in ternary blend bulk heterojunction organic solar cells. J. Am. Chem. Soc. 135, 986-989 (2013).

34. Kouijzer, S., Li, W., Wienk, M. M. \& Janssen, R. A. J. Charge transfer state energy in ternary bulk-heterojunction polymer-fullerene solar cells. J. Photon. Energy 5, 057203-057203 (2014).

35. Lu, L., Luo, Z., Xu, T. \& Yu, L. Cooperative plasmonic effect of Ag and Au nanoparticles on enhancing performance of polymer solar cells. Nano Lett. 13, 59-64 (2012).
36. Wu, J.-L. et al. Surface plasmonic effects of metallic nanoparticles on the performance of polymer bulk heterojunction solar cells. ACS Nano 5, 959-967 (2011).

37. Lu, L. et al. The role of $\mathrm{N}$-doped multiwall carbon nanotubes in achieving highly efficient polymer bulk heterojunction solar cells. Nano Lett. 13, 2365-2369 (2013).

38. Riedel, I. et al. Effect of temperature and illumination on the electrical characteristics of polymer-fullerene bulk-heterojunction solar cells. Adv. Funct Mater. 14, 38-44 (2004).

39. Cowan, S. R., Roy, A. \& Heeger, A. J. Recombination in polymer-fullerene bulk heterojunction solar cells. Phys. Rev. B 82, 245207 (2010).

40. Koster, L. J. A., Mihailetchi, V. D., Ramaker, R. \& Blom, P. W. M. Light intensity dependence of open-circuit voltage of polymer:fullerene solar cells. Appl. Phys. Lett. 86, 123509 (2005).

41. Lu, X. et al. Bilayer order in a polycarbazole-conjugated polymer. Nat Commun 3, 795 (2012)

42. Smilgies, D.-M. Scherrer grain-size analysis adapted to grazing-incidence scattering with area detectors. J. Appl. Crystallogr. 42, 1030-1034 (2009).

43. Chen, W., Nikiforov, M. P. \& Darling, S. B. Morphology characterization in organic and hybrid solar cells. Energy Environ. Sci. 5, 8045-8074 (2012).

44. Chen, W. et al. Hierarchical nanomorphologies promote exciton dissociation in polymer/fullerene bulk heterojunction solar cells. Nano Lett. 11, 3707-3713 (2011).

45. Jiang, Z. et al. The dedicated high-resolution grazing-incidence X-ray scattering beamline 8-ID-E at the advanced photon source. J Synchrotron Radiat. 19, 627-636 (2012).

46. Gann, E. et al. Soft x-ray scattering facility at the advanced light source with real-time data processing and analysis. Rev. Sci. Instrum. 83, 045110-045114 (2012)

\section{Acknowledgements}

This work is partially supported by US National Science Foundation grant (NSF CHE1229089, DMR-1263006), US, Air Force Office of Scientific Research and NIST ChiMad programme. W.C. gratefully acknowledges financial support from the US Department of Energy, Office of Science, Materials Sciences and Engineering Division. We also thank Dr Joseph Strzalka and Dr Cheng Wang for the assistance with GISAXS and RSoXS measurements. Use of the Advanced Photon Source (APS) at the Argonne National Laboratory was supported by the US Department of Energy, Office of Science, Office of Basic Energy Sciences, under Contract No. DE-AC02-06CH11357. The ALS at the Lawrence Berkeley National Laboratory is supported by the Director, Office of Science, Office of Basic Energy Sciences, of the US Department of Energy under Contract No. DE-AC02-05CH11231.

\section{Author contributions}

L.Y. conceptualized and supervised the project. L.L. carried out the fabrication and characterization of ternary blend devices and data analysis. T.X. conducted the synthesis of polymers. W.C. performed X-ray scattering experiments and analyses. All authors discussed the results and L.Y., L.L. and W.C. prepared the manuscript.

\section{Additional information}

Supplementary Information accompanies this paper at http://www.nature.com/ naturecommunications

Competing financial interests: The authors declare no competing financial interests

Reprints and permission information is available online at http://npg.nature.com/ reprintsandpermissions/

How to cite this article: Lu, L. et al. High-performance ternary blend polymer solar cells involving both energy transfer and hole relay processes. Nat. Commun. 6:7327 doi: $10.1038 /$ ncomms8327 (2015)

This work is licensed under a Creative Commons Attribution 4.0 International License. The images or other third party material in this article are included in the article's Creative Commons license, unless indicated otherwise in the credit line; if the material is not included under the Creative Commons license, users will need to obtain permission from the license holder to reproduce the material. To view a copy of this license, visit http://creativecommons.org/licenses/by/4.0/ 